\title{
Los árboles que esconden al bosque: Miguel Ángel de Quevedo y los orígenes de la ciencia forestal en México
}

\author{
JuAN Luis Delgado
}

\begin{abstract}
PALABRAS CLAVE: Quevedo, Estado, transferencia de conocimientos, bosques.
\end{abstract}

\section{CÓDIGOS JEL: N56, O14, Q23, Q28.}

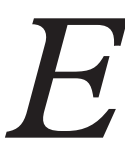

l presente artículo trata sobre el papel del ingeniero civil Miguel Ángel de Quevedo (1862-1946) en los orígenes de la ciencia forestal en México. El hilo conductor será determinar si las críticas de varios de sus contemporáneos a su proyecto sobre la atención preferente al árbol en lugar del bosque tenían o no fundamento. Se pondrá especial acento en los primeros encuentros entre el Estado y la ciencia forestal, en la configuración de las ideas forestales que sirvieron de guía para su desarrollo y, finalmente, en la formación de personal facultativo. Las principales fuentes de trabajo son los textos publicados por el mismo Quevedo. La conclusión es que el proyecto del ingeniero estuvo efectivamente más orientado hacia el árbol en lugar del bosque, sobre todo después de la Revolución mexicana. No obstante, a pesar de su marcada inclinación conservacionista, de sus carencias conceptuales, su escaso legado científico en matería silvícola y su franca inclinación hacia la hidrología forestal, es innegable que Quevedo fue el responsable de introducir y convertir la ciencia forestal en un asunto de Estado en México. Ante el peso sociopolítico de lo agrícola y de una deforestación endémica, no se puede considerar un aporte menor. 


\title{
The trees that hide the forest: Miguel Ángel de Quevedo and the origins of forestry in Mexico
}

\author{
KEYWORDS: Quevedo, state, knowledge transference, forests.
}

\section{JEL CODES: N56, O14, Q23, Q28.}

his article describes the role of civil engineer Miguel Angel de Quevedo
(1862-1946) in the origins of forestry in Mexico. The guiding issue is to de-
termine whether the critiques of some of his contemporaries regarding his project, which prioritised attention to the tree rather than the forest, were well-founded. Particular emphasis will be placed on the first encounters between the state and forestry, the development of ideas that guided the growth of forestry and the training of forestry workers. The principal sources are the texts published by Quevedo himself. The author concludes that Quevedo's project favoured the tree over the forest, especially after Mexican revolution. However, despite his conceptual shortcomings, his scant scientific legacy in silvicultural matters, his insistence on forest hydrology and his strong conservationist inclinations, Quevedo was undeniably responsible for introducing forestry science to Mexico and making it a state concern. Given the endemic deforestation and the sociopolitical weight of agriculture in Mexico, this cannot be considered a minor contribution.

Recepción: 2017-12-08 • Revisión: 2018-07-12 • Aceptación: 2018-07-25

Juan Luis Delgado [orcid.org/0000-0002-4555-4555] es investigador independiente egresado posdoctoral del Instituto de Geografía de la Universidad Nacional Autónoma de México. Dirección para correspondencia: Dr. Atl 39-8, Col. Santa María la Ribera 06400, Cuauhtémoc, Ciudad de México (México). C. e.:juanluisdelgado80@gmail.com 


\section{INTRODUCCIÓN}

La cuestión forestal se convirtió en un asunto de Estado en México gracias al trabajo y tenacidad del ingeniero civil Miguel Ángel de Quevedo (Guadalajara, 1862-Ciudad de México, 1946) ${ }^{1}$. Antes de él nadie había logrado integrar de forma definitiva los bosques en la agenda pública del gobierno federal, ni tampoco introducir postulados científicos para su manejo. Para varios de sus coetáneos, sin embargo, era claro que su proyecto no estaba encaminado hacia un aprovechamiento racional del bosque, sino a su conservación por sus atributos medioambientales. Algunos de sus críticos, como el empresario papelero Alberto Lenz (1867-1951) o el biólogo Enrique Beltrán (1903-1994), le reprocharon esta inclinación y, sobre todo, su atención al árbol en lugar de al bosque. El árbol no puede ser perenne, el bosque sí, sostenía el empresario (Lenz, 1990: 631).

La diferencia aparentemente sencilla entre el árbol y el bosque había pasado desapercibida para la aún incipiente historiografía forestal mexicana. Esta distinción permite realizar un recorrido distinto por la historia de los bosques en México. Mientras la gran mayoría de aquella historiografía se ha enfocado en el análisis de la intervención del Estado para gestionar los bosques ${ }^{2}$, aquí se parte del interés por estudiar la conformación de la rama científica forestal en su relación con el Estado ${ }^{3}$. Tanto las influencias extranjeras en la materia como su traducción a la realidad nacional por parte de Quevedo son objetos clave en este artículo. Dicha traducción, como se verá, se formuló y reformuló precisamente por las vicisitudes del Estado mexicano. Por lo tanto, este artículo no se aleja de la historiografía forestal, que, dentro y fuera de México, prioriza el papel del Estado en el establecimiento de la ciencia forestal, sino que propone profundizar en los aspectos científicos de esa historia ${ }^{4}$.

$\mathrm{Al}$ analizar la labor de Quevedo en la introducción de la ciencia forestal en México se busca mostrar cómo se fue desarrollando su proyecto durante una de las etapas más

1. Su nombre completo era Miguel Ángel García de Quevedo y Zubieta. Por razones aún desconocidas se dio a conocer públicamente como Miguel Ángel de Quevedo.

2. Simonian (1999); González Pérez (2001); Klooster (2003); Boyer (2005, 2007, 2015); BOYER y WAKILD (2012); WAKILD (2006, 2011); MATHEWs (2011); URQUIZA (2014); VARGAS (2017).

3. De los autores antes referidos, Mathews fue quien más penetró en los postulados científicos; sin embargo, lo hizo especialmente sobre la relación fuego-bosque en la comunidad de Ixtlán de Juárez, Oaxaca.

4. Fuera de México la historiografía forestal es profusa. Como botón de muestra, véanse AGNOLETTI (2006), quien aborda la historia de la historiografía forestal a nivel internacional, y también el reciente trabajo comparativo entre países del norte de Europa coordinado por OOSTHOECK y HöLZL (2018). 
convulsas de la historia de México: el primer tercio del siglo xx. De esta forma se podrá determinar si los reproches de Lenz y Beltrán tenían o no fundamento, es decir, si los árboles escondieron el bosque en el origen de la ciencia forestal en México. La forma de realizar este abordaje será principalmente a partir del análisis de los textos del propio Quevedo publicados en las revistas forestales mexicanas de la época. Específicamente tres serán los puntos que se tratarán: los primeros encuentros entre el Estado y la ciencia forestal, la configuración de las ideas forestales y la formación de personal facultativo.

\section{LA CIENCIA FORESTAL Y EL ESTADO: PRIMEROS ENCUENTROS}

Como en los Estados Unidos, los primeros intentos por introducir la ciencia forestal en México fueron respuesta a una realidad tangible: la deforestación. Durante las primeras décadas del México independiente, los diferentes gobiernos del país repitieron la fórmula novohispana ante la tala excesiva: replantar lo talado ${ }^{5}$. El problema era que se trataba de una respuesta poco meditada. Cualquier política en materia forestal estaba condenada al fracaso si desconocía la dinámica de sus bosques y, especialmente, si no disponía de un inventario forestal ${ }^{6}$. La única manera concreta que tenía el Estado para intentar remediar el problema era mediante la promulgación de leyes.

En octubre de 1894 se publicó el Reglamento para la explotación de los bosques y terrenos baldíos y nacionales. No era el primer reglamento sobre el tema, pero sí el más completo hasta la fecha. De esta normativa destacan tres puntos: 1) la formación de los agentes y encargados de vigilar la explotación; 2) la proyección de un inventario; y 3) la escasa referencia a postulados forestales. Sobre el primer punto, el reglamento nunca aludió al tipo de educación mínima necesaria de los agentes para desarrollar su labor, solamente se mencionaba que la práctica y la experiencia derivada del ejercicio de su trabajo los haría capaces de hacer mejores propuestas de manejo (Art. 3, fracción XI). Con respecto al segundo punto, se decía que las reglas pertinentes para explotar y asegurar la reproducción vegetal se dictarían cuando se conocieran las especies de árboles que, según la región, constituyan los montes nacionales, y el estado en que éstos se encuentran (Art. 27). En cuanto al tercer punto, los postulados forestales introducidos se redujeron a dictar pautas para

5. Sobre la política forestal mexicana del siglo xIX, URQUiZa (2014), TORRES (2017) y VERGARA (2018).

6. Dos intentos fracasados por inventariar las forestas del país tuvieron lugar en la segunda mitad del siglo XIX: el primero en 1868 y el segundo entre 1884 y 1889 (GARCía MARTínEZ, 1942: 236; EsTÉVEZ, 1956: 141-142). 
cortar o resinar únicamente árboles adultos, así como someras indicaciones para la repoblación: por semilla en el monte alto y por brotes en el bajo (Art. 26).

Es importante reconocer en este reglamento la influencia española en tres sentidos: 1) en la utilización del vocablo monte en lugar de bosque ${ }^{7}$; 2 ) en su categorización como montes altos y bajos (los primeros haciendo referencia a los maderables y los segundos a todos los demás); y 3) en la experiencia como base para la acción forestal.

El origen de esta influencia es todavía desconocido, pues las tres publicaciones de la Secretaría de Fomento antes de 1894 no se enfocaban en los montes, sino en los bosques ${ }^{8}$. En el mismo título lo indican: Memoria sobre la utilidad de los bosques (1873), del ingeniero Gabriel Hinojosa; Utilidad de los bosques (1882), del químico Eugenio Frey; y Selvicultura. Breves consideraciones sobre explotación y formación de los bosques (1892), del botánico, naturalista y geólogo Mariano Bárcena ${ }^{9}$. A pesar de que en estas obras la concepción de los bosques era la de espacios arbolados con funciones medioambientales, la vinculación entre las ramas agrícola y forestal era aún muy fuerte. Hinojosa, por ejemplo, declaraba que la silvicultura era una rama de la agricultura (cit. en Urquiza, 2014: 94). Bárcena, por su parte, proponía la mezcla de ambos usos de suelo allá donde fuera posible. En cuanto al asunto propiamente silvícola, el botánico expuso de forma somera los métodos de corta por entresaca o poda, y, aunque recomendó utilizar especies autóctonas para repoblar o restaurar los bosques con relación al clima y al suelo, la promoción del eucalipto fue profusa.

Ninguno de estos tres autores, sin embargo, hizo alusión al tema del personal facultativo, ni mucho menos a la experiencia necesaria para efectuar su labor. En España, al contrario, los llamados ingenieros de montes consideraban fundamental, desde 1848, la adquisición de experiencia, y tenían un amplio sentido histórico proyectado hacia al porvenir, es decir, hacia el progreso (Delgado, 2015: 225). Por su parte, en México, a pesar

7. El ingeniero de montes español Agustín Pascual aclaró en 1855 la diferencia entre las voces monte y bosque en el tomo 4 del Diccionario de Agricultura Práctica y Economía Rural. El primero, que era propiamente el campo de acción de los forestales, era una extensión de tierra cubierta por plantas silvestres y espontáneas, mientras que el segundo era un sitio poblado de árboles y matas espesas donde se crían los animales propios para la caza.

8. A principios de la década de 1880 hubo un intento fallido de la Secretaría de Fomento por traducir obras alemanas de arboricultura (URQUIZA, 2014: 110).

9. Este folleto se distribuyó en julio de 1892 a los diferentes estados y territorios de la República para su empleo como instructivo. Por eso, hay quienes consideran a M. Bárcena el precursor de la enseñanza forestal en México (García Martínez, 1942; Caballero, 2000). Por otro lado, es importante mencionar que el término selvicultura es de uso más común en España, mientras que el de silvicultura lo ha sido en Hispanoamérica (GONZÁLEZ-DonCEL \& GIL, 2013). 
de que la preocupación por los bosques apuntaba igualmente hacia el futuro, la ejecución del reglamento de 1894 era irrealizable por un motivo: la inexistencia de un cuerpo de facultativos capacitados para ordenar la experiencia.

En el último tercio del siglo XIx, la única persona que indicó la necesidad de formar facultativos fue el alemán Isidoro Epstein (Urquiza, 2014: 129). El abogado Ricardo Ramírez y el botánico Georges Lapie aludieron de forma tangencial dicho asunto cuando refirieron las causas del fracaso del citado reglamento. Según ellos, los fallos fueron la escasez de vigilancia, así como de medios para sufragarla, la inclinación al cohecho del personal encargado de hacer valer el reglamento (debido a su exiguo sueldo y a su condición de subalternos), la inexistencia de un inventario de bosques nacionales y, finalmente, que la Secretaría de Fomento centraba sus trabajos en los alrededores del Distrito Federal, dejando al arbitrio de los gobiernos locales la gestión de sus respectivos bosques (Ramírez, 1897: 28; Lapie, 1912: 620) ${ }^{10}$.

En este sentido, no sorprende la convocatoria realizada en 1894 por la Secretaría de Fomento, a través de la Sociedad Agrícola Mexicana, para premiar a la mejor obra de silvicultura en la que se dé preferente lugar en su estudio, cultivo y explotación a las especies indígenas, más bien que a las exóticas [...], puesto que sobre tan importante materia, y aplicable a nuestro país, poco o nada hay escrito que pueda considerarse como un buen cuerpo de doctrina (cit. en Urquiza, 2014: 135).

La llegada de Miguel Ángel de Quevedo a la Administración pública porfiriana empezó a modificar esa superficial ocupación del Gobierno por la vida de los bosques. Quevedo había regresado a México en 1888 luego de concluir su formación en París como ingeniero de caminos y puentes. La conservación de los bosques fue ocupando su interés mientras trabajaba en la instalación de algunas plantas hidroeléctricas en la década de 1890. Ahí fue donde se dio cuenta del lamentable estado de las zonas arboladas que rodeaban las cuencas hidrológicas, y ahí fue donde empezó a aplicar los conocimientos en hidrología forestal que había aprendido en Francia.

10. Algunos autores contemporáneos han destacado que el proyecto forestal encabezado por Quevedo se caracterizó por su centralismo al olvidarse del resto de los bosques del país (especialmente, de los tropicales), y también por concebir la idea de lo forestal desde la ciudad, al considerar la necesidad de los bosques en relación directa a la higiene pública de las urbes (MATHEws, 2011; VITZ, 2012; BOYER, 2015). Otros autores, al contrario, destacan el hecho de que se contemplaba el problema en su complejidad higiénica, agrícola, industrial, energética, biológica y urbanista, por tanto, la implantación de esta ciencia no podía entenderse en otras condiciones que no fueran las del valle de México (CASAls, 1995; URQuiza, 2014). 
Las ideas de Quevedo, por tanto, encajaron con la concepción del bosque como protector medioambiental ${ }^{11}$. Así, la influencia española contenida en el reglamento de 1894 fue relegada para dar lugar a los que se podrían considerar los pilares del primer proyecto forestal mexicano, a saber: la pretensión de contribuir a erradicar la deforestación, promover la higiene pública - de las urbes-, suministrar recursos para las industrias y, como corolario esencial, sostener la idea de que la falta de árboles era causante de la disminución en el régimen pluvial, del aumento de las inundaciones, del empobrecimiento del suelo, de los declives en los flujos hídricos y de las variaciones en el clima ${ }^{12}$. Todo esto se lograría mediante la conservación y repoblación, siempre con un enfoque utilitario en sentido biológico y económico, en el presente y hacia el futuro.

De tal forma, gracias a la tenacidad y persistencia del ingeniero tapatío nació en septiembre de 1904 la primera institución mexicana dedicada exclusivamente al tema forestal: la Junta Central de Bosques (JCB, en adelante), correspondiente al Distrito Federal y dependiente de la Secretaría de Fomento ${ }^{13}$. Quevedo había logrado convencer de la necesidad de tomar cartas en el asunto de los bosques a personajes clave de la vida política y económica del régimen porfirista.

Sin embargo, la JCB no empezó realmente a ejecutar su programa hasta 1907. Con apoyo del secretario de Hacienda, JoséY. Limantour, y del nuevo secretario de Fomento, Olegario Molina, entre 1907 y 1910 comenzó a conformarse el Servicio Forestal mexicano al instituirse el Vivero de Coyoacán (en terrenos donados por el propio Quevedo) y las escuelas para guardas forestales de Coyoacán y Santa Fe, en los alrededores del Distrito Federal (Quevedo, 1909b: 8-9; Hickel, 1910: 149).

El proyecto forestal, no obstante, recibió un duro revés en el mismo año de 1910 al suprimirse la JCB. En opinión de Quevedo, esto se debió a las presiones de varios agrónomos. En su lugar se creó el Departamento de Bosques, dependiente de la Dirección General de Agricultura. Esta modificación institucional restaba capacidad de acción y supeditaba el programa forestal a las prioridades agrícolas. Como contrapartida, se había aprobado la creación de la carrera de ingeniero de montes para impartirse en la Escuela Nacional de Agricultura (ENA, en adelante). Pero esto no era suficiente para Quevedo.

11. Las ideas de Quevedo conectaban en especial con la obra de Frey, pues este abordaba el asunto desde la perspectiva hidrológico-forestal.

12. Se trataba de una idea vieja nombrada por Humboldt como teoría de la desecación, la cual, no obstante, ha sido desde entonces y hasta la fecha objeto de controversia (ANÓNIMO, 1897: 35-36; MATHEWS, 2011: 40; URQUIZA, 2014: 100; BoYeR, 2015: 53).

13. En diciembre de ese año empezaron a constituirse las juntas locales de bosques en Aguascalientes, Guadalajara, Morelia, Oaxaca, Toluca y Zacatecas (URQUIZA, 2014: 223). 
Para él, era fundamental mantener una institución forestal autónoma de otras dependencias de gobierno, sobre todo de las agrícolas. En la práctica dicha carrera no se materializó por el mismo desinterés que en el medio agrícola se tiene por la cuestión forestal (Quevedo, 1927: 72), lo cual provocó que la educación tuviera que ser solamente para formar guardas y no ingenieros. En una misiva de julio de 1910 dirigida a Limantour, Quevedo solicitaba su ayuda para recuperar la autonomía institucional; pero el secretario de Hacienda se encontraba fuera del país y no pudo asistirlo. Aun estando, quizá nada hubiera podido hacer, pues, como el mismo Quevedo le confesaba, había perdido el apoyo del secretario de Fomento posiblemente por sus ideas al respecto de una legislación que restringiera el libre uso de los bosques particulares y municipales ${ }^{14}$. Meses después estalló la Revolución mexicana.

El hecho más relevante de esta primera etapa fue la ratificación del Estado como el mayor responsable en la institución y desarrollo de una ciencia aplicada a la gestión de los bosques. Los sucesivos gobiernos revolucionarios, desde Madero hasta Cárdenas, con la excepción de Huerta, estuvieron siempre en la disposición de al menos escuchar las preocupaciones por la conservación forestal. El apoyo efectivo brindado a esta causa fue, sin embargo, muy desigual. Asimismo, si ya antes de 1910 el interés agrícola era absoluto en términos económicos y educativos, después de esa fecha se volvió una de las piedras angulares del proyecto de nación. La aplicación de los postulados forestales encabezados por Quevedo, aunque ya no tenía que lidiar con el respeto absoluto a la propiedad privada y a la Constitución liberal de 1857, ahora lo debía hacer con respecto a las formas que estaba adoptando el reparto de tierras. A partir de entonces se agudizó la histórica confrontación entre campos y bosques, pues la Constitución de 1917 contemplaba en el famoso artículo 27 la obligación de repartir tierras y de procurar la conservación de todos los recursos naturales de la nación.

En síntesis, desde una honda preocupación por detener la deforestación, el origen de la primera ciencia forestal en México se topó con la omnipresencia de lo agrícola. Como se verá a continuación, esta circunstancia contribuyó a remodelar el proyecto de Quevedo luego de la revolución.

14. Centro de Estudios de Historia de México (CEHM), archivo Limantour, fondo CDLIV. carpeta 24, legajo 57. Carta de Miguel Ángel de Quevedo a JoséY. Limantour, 10 de julio de 1910. 


\section{CONFIGURACIÓN DE LAS IDEAS FORESTALES}

En la inauguración del primer Congreso Forestal Nacional, celebrado en la Ciudad de México en febrero de 1930, Quevedo señaló que, a pesar de los trabajos emprendidos desde hacía más de veinte años, la intensa destrucción forestal había continuado. Por ejemplo, decía, en 1910 los bosques correspondientes al Distrito Federal abarcaban 22.000 hectáreas, pero para entonces quedaban únicamente 7.000. Así las cosas, la labor conservacionista implicaba aún más esfuerzo: ante tal panorama, sostenía Quevedo, mayor era la responsabilidad mexicana frente a las demás naciones, pues la conservación en el concepto mundial humanitario no tiene fronteras (Quevedo, 1930a: 31-33).

El sentido transnacional del proyecto forestal de Quevedo había aflorado desde 1909 cuando participó como representante del Gobierno mexicano en el Congreso sobre Conservación de Recursos Naturales en Norteamérica, celebrado en la ciudad de Washington. Este congreso causó un hondo efecto en él, pues en ningún momento de su vida pública como forestal olvidó los paradigmas expuestos en la capital norteamericana. Ahí se estableció como preponderante el elemento bosque, equilibrador de los demás e indispensable de conservarse en grandes extensiones del territorio de cada nación, recomendándose constituir a ese fin extensas Reservas Forestales [...], sometidas a un régimen de explotación muy restringida para su indefinida conservación (Quevedo, 1934: 106). De tal forma, la conservación se mantuvo como el principal objetivo para Quevedo desde el inicio (1904) hasta el final de su carrera pública forestal (1940). Su proyecto se basaba en un determinismo geográfico que creía inalienable la influencia del bosque sobre el clima, las precipitaciones, la hidrología y el suelo.

La experiencia de Quevedo en hidrología forestal, así como su insistencia en la necesidad de impulsarla, condicionaron su proyecto y su proyección internacional. Por ejemplo, en el Congreso de 1909 se le recordó más por el acento dado a los bosques sobre la regulación de las aguas que por su capacidad para producir madera (Barthod, 2015: 5861, 66). Tal como ha señalado en su tesis doctoral Juan Humberto Urquiza (2014), la persistencia de Quevedo en la regeneración de las cuencas hidrológicas era el fundamento de su proyecto conservacionista ${ }^{15}$.

A diferencia de su contraparte norteamericana, el famoso forestal Gifford Pinchot (1865-1946), quien sostenía la necesidad de revitalizar en cada generación las nociones

15. Sin embargo, creo necesario no dejar de tener en cuenta la estrecha relación de Quevedo con empresas hidroeléctricas en las cuales él mismo tenía participación accionaria (véanse QuEVEDO, 1943; PACHeCo, 2017). 
sobre conservación, Quevedo se refirió a este concepto casi siempre en los mismos términos, a saber: por sus beneficios biológicos y económicos. No obstante, luego de la revolución integró en su definición las utilidades estéticas, incentivadas con la creación de parques nacionales (Wakild, 2011), y las sociales, subordinadas al patriotismo de ciudadanos y campesinos (Boyer, 2015). Para el Quevedo de los años veinte y treinta, la conservación y la patria eran un binomio inexorable. Según él, en todas las naciones cultas el exponente más característico de la propia cultura es la conservación de los bosques [...]. $Y$ es que las leyes mismas de la Naturaleza, puestas de manifiesto por la experiencia de siglos de la vida humana en el Planeta, asi lo exigen. Precisamente, uno de sus modelos eran los Estados Unidos, donde para popularizar y hacer más comprensivo y utilitario el movimiento de protección a la naturaleza se utilizó el término conservación en un sentido patriótico, de verdadera conservación nacional (Quevedo, 1934: 105-106).

Los paradigmas propiamente forestales se dieron a conocer en México con el trabajo La Dasonomía: generalidades, publicado por la Secretaría de Fomento en 1903. Esta obra fue elaborada en 1895 por el ingeniero agrónomo Manuel R. Vera con motivo de la ya mencionada convocatoria realizada por dicha Secretaría para premiar a la mejor obra de silvicultura. Su impacto, sin embargo, fue mínimo. El término dasonomía, por ejemplo, que es el nombre técnico de la ciencia forestal, apenas se lee en los textos forestales de los siguientes cuarenta años. La dasonomía, según la definió Vera apoyado en ingenieros de montes españoles como Agustín Pascual (1818-1884) o Lucas de Olazábal (1829-1899), y en el forestal alemán Heinrich Cotta (1763-1844), es el conjunto de principios y reglas para la cría, conservación y aprovechamiento de los montes, y está formada a su vez por tres ramas: la dasótica, que tiene por objeto la repoblación y conservación natural de los bosques, aprovechando los beneficios que resultan de la recolección de los productos; la silvicultura, dedicada a la formación de los bosques por procedimientos artificiales; y la $d a-$ socracia, aplicada a ordenar el aprovechamiento de los montes, regularizando la producción para obtener un rendimiento anual igual y constante (Vera, 1903: 5-7).

En México, no obstante, el término para referirse a la ciencia forestal fue el de silvicultura. La experiencia francesa de Quevedo fue determinante en esta elección epistémica. Así, a pesar de que en más de un sentido la ciencia forestal en Francia ha sido tributaria de la dasonomía alemana, según un artículo de Ph. Guinier publicado en el primer número del Boletín del Departamento Autónomo Forestal en 1935, en Francia la silvicultura era la cabeza de las Ciencias Forestales [...] cuyo objeto es definir los medios de asegurar la perpetuidad y prosperidad del bosque (Guinier, 1935: 198). Al reproducir esta concepción, dicho boletín (dirigido por Quevedo) ratificaba la distinción silvícola sobre la dasonómica. En esos años, Guinier era director de la Escuela Nacional de Aguas y Bosques, y según declaraba en el mismo texto, un fuerte movimiento de opinión se había generalizado en 
Francia a favor de los bosques, pues se había reconocido su gran utilidad económica en la regularización de las aguas. Sin embargo, el francés era plenamente consciente de que ese aspecto era solo uno más en los que debía intervenir la silvicultura. Quevedo, al contrario, vitoreaba la vieja decisión francesa de reunir en una sola corporación facultativos dedicados a gestionar la relación entre las aguas y los bosques: fomentar el vínculo entre las ramas hidráulica y silvícola era prioritario para él (Quevedo, 1934: 111).

En cualquier caso, el gran problema era cómo ejecutar el objetivo de instaurar paradigmas científicos foráneos ${ }^{16}$. La forma de ejecución depende de las ideas que la dirijan. En el caso que nos ocupa estas ideas apenas se estaban creando. De los ya referidos beneficios biológicos y económicos de la conservación del bosque, en la práctica se dio mayor atención al primer aspecto. Una posible explicación sería la falta de personal facultativo para asesorar, conducir y vigilar el aprovechamiento. Pero el asunto es más complicado. Sabemos que los procesos de transferencia de nuevos conocimientos a un determinado entorno social son complejos y en ocasiones hasta traumáticos (Casals, 1995: 607); es decir, ni para Quevedo ni para ningún afiliado a la causa debió ser sencillo hacer ver a los legos en la materia un tipo de conocimiento y de práctica que posiblemente ni siquiera ellos mismos entendían del todo. Ninguno de los mexicanos que se unieron a Quevedo era forestal de profesión; sus conocimientos podían girar en mayor o menor medida en torno al bosque, lo cual era necesario para advertir sus elementos constitutivos, pero no suficiente para adaptar postulados silvícolas ${ }^{17}$. De ahí la incapacidad de gestionar el aprovechamiento, que también se podría plantear en la atención al árbol en lugar de al bosque.

En sus inicios el proyecto de la JCB iba dirigido a los bosques (concepto este que, sin embargo, nunca quedó definido ni por Quevedo ni por nadie que lo secundara). Sin embargo, por el contacto de esta institución con otras autoridades y con la ciudadanía en general tuvo que derivar hacia el repoblamiento de árboles en las ciudades, es decir, hacia la arboricultura.Y como el desconocimiento en la materia era igualmente sustancial, esta nueva inclinación encajó a la perfección en el programa de la JCB al extender nuestra acción a todo lo que sea protección y fomento del árbol, pues siendo que este es el rey de la vegetación, y nuestra labor tiende a que no falte en parte alguna del territorio la cubierta vegetal del suelo, que es el ideal del forestal (Redacción, 1909: 29-32; cursivas en original).

16. HiCKel (1913: 15) señaló que la traducción al español de la obra Silvicultura, de G. Lapie, un folleto escrito en 1910 exclusivamente para las escuelas mexicanas, tenía errores de consideración; por ejemplo, el término peuplements (plantaciones) se tradujo como clasificación de bosques.

17. Entre los mexicanos que participaron del proyecto en un inicio estaban los ingenieros agrónomos Gilberto Serrato, Higinio Zúñiga, Waldo Cienfuegos, Tereso Reyes y el citado Manuel R. Vera, el botánico Cándido de la Fuente, y el médico y naturalista Francisco Valenzuela. 
Desde entonces el nombre institucional cambió al de Junta Central de Bosques y Arbolados. De esta manera el ideal del forestal se focalizó en el rey de la vegetación, es decir, en el cultivo de árboles, cosechando los mayores éxitos del proyecto en este sentido, sobre todo en el Distrito Federal y sus alrededores ${ }^{18}$. Según G. Lapie (1912: 620), elVivero de Coyoacán, que entregaba las plantas gratuitamente, tuvo éxito en la producción de árboles frutales y de ornato, pues las especies forestales despertaron escaso o nulo interés.

A pesar de ello, el programa de aprovechamiento silvícola de la JCB no se abandonó. Al menos, teóricamente. En el catálogo de bosques mexicanos publicado en 1911 se registraron principalmente las especies maderables: las de montaña, de llano y de ribera, las de parques y calzadas, e incluso las de arbustos siempre que [...] proporcionen productos leñosos aprovechables en la industria (Quevedo, 1911: 126) ${ }^{19}$. Por una parte, esto quiere decir que no se registró toda la vegetación espontánea de los montes (lo que algunos autores foráneos consideraban como especies propiamente forestales), mientras que, por la otra, nos confirma una concepción utilitaria del vocablo forestal.

No fue hasta los años veinte cuando Quevedo expresó públicamente su definición de lo forestal, aunque más estimulado por la necesidad de delimitar esa clase de terrenos frente a los agrícolas que por aclarar científicamente su contenido. Primero dijo que la riqueza forestal comprende todos los productos espontáneos del suelo, sean pastizales, arbustos, chaparrales, montes bajos y bosques; luego explicó que, desde los romanos, la foresta, proveniente del término latino foras (fuera), es la vegetación espontánea que está fuera del poblado y del campo agrícola, quedando el adjetivo forestal como la referencia a esa vegetación; finalmente puntualizó, por un lado, que los terrenos forestales son inadecuados para el cultivo agrícola y, por el otro, que los cultivos forestales son más rústicos que los agrarios, lo que hace que los métodos de explotación o cosecha sean también distintos ${ }^{20}$.

En La Cuestión Forestal en México, folleto publicado en 1909 y dirigido al secretario de Fomento, Quevedo exponía algunas de estas ideas dentro de lo que consideraba la prin-

18. A Quevedo tampoco le costó trabajo integrar la arboricultura orientada a la ciudad. Desde 1901 tenía esas ideas en mente cuando participó en el Primer Congreso Internacional de Higiene Pública y Problemas Urbanos en París. Una de sus acciones más relevantes fue aumentar la cobertura forestal de la Ciudad de México en un 16\% para 1910, pero además haber popularizado la idea de conservación forestal entre la clase urbana educada y haber creado un espacio para el tributo público al árbol (WAKILD, 2006: 7-8, 11).

19. Aunque este catálogo fue el primer intento serio por inventariar la floresta forestal nacional, fue todavía demasiado general, impreciso y, lo más importante, poco operativo. Por otra parte, Quevedo reconoció que la JCB intensificó los trabajos en este sentido por la congoja de no haber podido presentar datos estadísticos en el Congreso de Washington de 1909 (QUEVEDO, 1919: 151).

20.Quevedo (1925a: 113; 1926: 131; 1927: 73; 1930b: 73-5). 
cipal cuestión forestal, a saber: la mediación del Estado para someter a un régimen forestal los bosques del país, demarcándolos según su propietario, normando y vigilando lo que en cada uno debía permitirse, limitarse o prohibirse ${ }^{21}$. Quevedo fue insistente también en el hecho de que los bosques, independientemente de su propietario, debían ser aprovechados, pues solo la ordenada explotación constituía para el mismo bosque su higiene, prosperidad, y la mejor garantía para su conservación: el bosque debía tratarse como un capital cualquiera, decía ${ }^{22}$.

Por lo mismo, era importante tener una definición más o menos clara de la ciencia forestal. Para Quevedo, era una fusión entre: 1) conocimientos de las ciencias físicas y naturales aplicadas a la parte cultural del bosque o silvicultura, para que aquél indefinidamente se renueve y produzca los mayores rendimientos; 2) conocimientos de las ciencias matemáticas y mecánicas aplicadas a la Ordenación Forestal para estimar las existencias, su desarrollo, y aplicar los métodos más apropiados para la extracción y regeneración; y 3) química aplicada, para el más completo aprovechamiento de todos los componentes (Quevedo, 1929: 137).

Sin embargo, Quevedo parece haber sido consciente de que en la práctica los elementos de la ciencia forestal eran difíciles de cumplir. Así, con respecto a la regeneración del bosque dijo en 1924 que no era conveniente atenerse como procedimiento preferente a las repoblaciones artificiales, costosas e inseguras, sino a las cortas de aclareo o entresaca, es decir, mediante un trabajo estrictamente silvícola, siempre sujetando las explotaciones a un régimen forestal bien estudiado para cada caso mediante planes provisionales al proyecto definitivo de ordenación. De la misma manera, en 1930 recomendó que las plantaciones planeadas en los sistemas nacionales de riego solo se efectuaran donde la destrucción vegetal era total, de lo contrario, se debía buscar la manera de favorecer la repoblación natural ${ }^{23}$. Así pues, al seguir esta línea de pensamiento, la pregunta que surge es la siguiente: ¿para qué entonces las plantas forestales producidas en el vivero?

21. Según el tipo de propietario, los bosques estaban clasificados de tres maneras: de la nación, que debían ser los más numerosos porque daban mayor garantías para la conservación (el Estado como mejor garante de los bosques fue un paradigma forestal alemán de enorme alcance); de municipios o comunidades, que exigían mayor participación de las autoridades locales; y de particulares, a los que ya no se les podía seguir juzgando como intocables por las leyes de utilidad pública (QUEVEDO, 1909a: 34-41).

22. Es poco probable que Quevedo conociera el famoso artículo del ingeniero de montes Agustín Pascual sobre el vocablo forestal (1868), donde precisamente argumenta que un monte se convierte en foresta cuando se le aplica trabajo y capital.

23. Quevedo (1924: 109); CEHM, archivo Antonio Carrillo Flores, fondo CCCLXII. 8. 6. 22. 
Como sea, al leer estos extractos de los textos del ingeniero antes y después de la Revolución, uno no puede tener la sensación de que se tratara de una persona que defendía una idea romántica de protección al árbol, como el biólogo Enrique Beltrán le reprochara (Beltrán, 1964). Paradójicamente, cuando en los años treinta Quevedo volvió a tener capacidad política, al quedar al frente del Departamento Autónomo Forestal y de Caza y Pesca, una parte de su proyecto se orientó hacia un conservacionismo radical al proclamar vedas a la explotación maderera en varios estados de la República. Incluso, cuando el 1 de enero de 1935 Lázaro Cárdenas anunció la creación del citado Departamento fue explícito al referirse al aprovechamiento maderero: únicamente se permitiría cuando obedezca a un fin social y siempre que redunde en provecho colectivo (Cárdenas, 1935: 2). A la postre, solo la extracción de resina de pino fue permitida, siempre y cuando se ejecutara siguiendo el método francés de resinación, pues se consideraba el más apropiado para lograr la conservación frente a los métodos americano o mexicano.

La promoción del aprovechamiento resinero quedó patente desde el primer año de la revista México Forestal (1923). Esta publicación, que reconocía explícitamente la influencia de la revista España Forestal y de la norteamericana fournal of Forestry, fue el órgano de difusión de la Sociedad Forestal Mexicana (SFM, en adelante), creada en 1921. Tanto la SFM como México Forestal fueron fundadas a iniciativa del propio Quevedo, de las cuales fue presidente y director, respectivamente. Ambas marcaron un punto de inflexión en la historia forestal mexicana, y aunque ninguna de las dos estaba vinculada directamente al Gobierno, su influjo en la modelación de las líneas forestales dominantes de los años veinte y treinta fue contundente.

La más clara fue su influencia en ley forestal de 1926 y en su reglamento del año siguiente. Los conceptos utilizados, por ejemplo, además de escasos, reflejaban en gran medida la autoridad de Quevedo. Así, el eje conceptual de la ley giró en torno a la idea de vegetación forestal, definida en el artículo 5 como aquella que al desarrollarse en un terreno es capaz de formar una cubierta que proteja al suelo contra los agentes de degradación y desecación. Es muy ilustrativo también que los terrenos forestales comunales o ejidales fueran definidos por negación al decir que son cualquier extensión de tierra que por sus condiciones naturales sea impropia para cultivo agrícola (Art. 14), mientras que un terreno privado se consideraría forestal cuando en él fuera posible la vida de los vegetales superiores y que por su localización, clima, topografía, calidad o condiciones económicas, dicho terreno no deba ser objeto de cultivos agrícolas (Art. 21). Al igual que esto sugiere la intención de ganar espacios para la reforestación, también parece celebrar el fin del respeto irrestricto a la propiedad privada, es decir, manifiesta la presencia de Quevedo ${ }^{24}$.

24. Por las implicaciones que tuvo sobre el futuro de comunidades que vivían del bosque, el artí- 
Pero volviendo a México Forestal y la SFM, es importante mencionar que varios de los miembros de esta última estaban vinculados a grandes empresas productoras y consumidoras de recursos madereros, especialmente compañías papeleras como Loreto y Peña Pobre, del empresario Alberto Lenz. Sin embargo, los artículos publicados en su revista no abordaron esta cuestión. La línea editorial estaba orientada hacia el estudio de árboles y bosques y casi nada al aprovechamiento, con excepción de la resina de pino (Cuadro 1).

\section{CUADRO 1}

Temas de la selección bibliográfica realizada por el Departamento Autónomo Forestal y de Caza y Pesca en 1939 sobre artículos publicados en revistas forestales mexicanas en 1909-1937

\begin{tabular}{lclc}
\hline Tema & Cantidad & \multicolumn{1}{c}{ Tema } & Cantidad \\
\hline Especies arbóreas & 25 & Ordenaciones (dasocracia) & 2 \\
Bosques específicos & 22 & Instituciones forestales & 2 \\
Bosques y aguas & 10 & Conservación de recursos & 2 \\
Aprovechamiento de resina & 9 & Incendios & 1 \\
Aves & 5 & Entomología & 1 \\
Estadística forestal & 4 & Dendrometría & 1 \\
Legislación & 4 & Cartografía & 1 \\
Plagas & 4 & Parques nacionales & 1 \\
Botánica & 3 & Suelos & 1 \\
Viveros & 2 & Fauna & 1 \\
Especies cactáceas & 2 & Vías de transporte & 1 \\
Estaciones forestales & 2 & Otros & 6 \\
TOTAL & & & 112 \\
\hline
\end{tabular}

Fuente: elaboración propia a partir de Redacción (1939: 219-231).

Se podría argumentar que las razones de esta orientación obedecieron a un interés genuino por conocer primero la ubicación y características de la floresta forestal mexicana. Quevedo (1911: 125), incluso, era claro al respecto: mucho se había escrito sobre plantas agrícolas y medicinales y poco o nada sobre las especies forestales, a pesar de su comprobada importancia económica. Importancia económica que no quedó reflejada en la

culo 16 de esta ley ha sido muy destacado por la historiografía forestal mexicana. Ahí se decretaba que la explotación comercial de terrenos comunales o ejidales solamente se podría realizar mediante cooperativas formadas por vecinos del lugar. BOYER (2015) afirma que esto fue la conformación de una ciencia forestal revolucionaria. Habría que seguir investigando el influjo de Quevedo en este punto. Varios estudios de caso se pueden encontrar en KLOOSTER (2003), MATHEWs (2011), Boyer (2005, 2015), WAKILD (2011), BOYER y WAKILD (2012). 
publicación de artículos sobre producción maderera. Para otros forestales, sobre todo los relacionados con la industria, esta circunstancia no pasaba desapercibida, y aunque no criticaban de manera frontal la autoridad de Quevedo, sí hacían mención a aspectos relevantes que era importante tener en cuenta. Así, por ejemplo, en 1930 el ingeniero de montes español Eduardo García, como miembro de la Compañía de Fábricas de Papel San Rafael $^{25}$, expuso en el marco del primer Congreso Forestal Nacional lo siguiente:

Pero esta obra de repoblación necesita, de todos modos de la cooperación del Estado, porque la Ciencia Forestal ni se improvisa, ni se importa. Podrán importarse ciertos conocimientos forestales generales, que no son suficientes para resolver el problema; podrán emprenderse con éxito algunas repoblaciones, y podrán también hacerse con éxito algunas determinadas ordenaciones, pero estos hechos serán la excepción, y la buena voluntad de las empresas se estrellará ante la falta de una Ciencia Forestal propia que garantice el empleo de los capitales [...]. Es, por consiguiente, indispensable que el Estado proporcione a las empresas particulares un personal técnico debidamente preparado, ya que este personal tiene que ser hijo del esfuerzo y de la experiencia de muchos años (García Díaz, 1930: 281).

Por su parte, cuando a principios de los años cuarenta el ingeniero forestal Camilo del Moral había dejado de trabajar en el Servicio Forestal (en el área de investigación silvícola) y formaba parte de la papelera San Rafael, criticó frontalmente la política forestal de Quevedo. Para Del Moral, era necesario borrar el concepto de inviolabilidad del árbol y de las masas forestales (Compañía de Fábricas de Papel..., 1942: 228).

Sin embargo, antes de la Revolución las tareas forestales emprendidas por la JCB poco tenían que ver con las críticas de Camilo del Moral. En la Revista Forestal Mexicana (1909-1911) se publicaron los resultados de los trabajos que la Sección de Estadística estaba realizando para la conformación de la base forestal mexicana, a saber: el registro de los recursos disponibles mediante inventarios y mapas ${ }^{26}$. Esta labor, suspendida en 1913, se retomó durante el sexenio cardenista, aunque sin resultados relevantes (Quevedo, 1919: 151; Boyer \& Wakild, 2012: 96).

25. La historia forestal de esta compañía es un magnífico tema de investigación por realizar. Por ejemplo, habría que profundizar en las relaciones de Quevedo con sus directores técnicos, los ingenieros de montes José de la Macorra y el citado Eduardo García, y los posibles beneficios que este vínculo podría haber reportado a la compañía. Para WAKILD (2011: 79), el apoyo del Gobierno a esta empresa representaba el interés del Estado por involucrarse en la economía de los recursos naturales.

26. El catálogo de 1911 antes citado, así como los mapas de las secciones forestales en que se dividió el Distrito Federal son la muestra de ello. 
Los problemas para dar una continuidad mínima al inventario forestal se manifestaron en diversos frentes. Los problemas políticos, institucionales y de dotación de personal han sido los más reconocidos, pero también los provocados por la topografía (sobre todo la dificultad para acceder a los bosques, conocer sus características y clasificar sus especies). Así, los trabajos publicados en las revistas forestales mexicanas sobre especies arbóreas y bosques específicos contribuyeron, sin duda, a paliar la enorme carencia en la literatura científica del país ${ }^{27}$. Sin embargo, todavía a principios de los años cuarenta no había obras de referencia, como sostenía el forestal suizo Arthur Meyer; aunque él mismo era consciente de que una descripción completa de los bosques de México era difícil por la variada topografía y porque las fuertes diferencias de altitudes correspondían a cambios en las condiciones climáticas y, por consiguiente, a los tipos de vegetación (Meyer, 1942: 55).

Para Quevedo, en cambio, la enorme variedad vegetativa derivada de las diversas altitudes y climas era la prueba de que México necesitaba una protección especial para sus bosques, pero también de que, bajo el aspecto forestal, lo hacía el territorio más interesante del globo (Quevedo, 1909a: 15; 1911: 129; 1936b: 212).

Meyer también hizo alusión a los problemas para determinar el nombre científico de importantes árboles forestales ubicados en regiones remotas del país. Quevedo, por su parte, decía que la problemática en la clasificación botánica de las especies era consecuencia de que en muchas partes del país se aplicaba el mismo nombre vulgar a diferentes especies. Es decir, el ingeniero no planteaba esta dificultad a partir de la topografía, sino a partir de las costumbres locales, revelando así de manera implícita lo que en muchas ocasiones, sobre todo después de la Revolución, hizo explícito: la baja estima que tenía sobre la población rural.

De tal forma, al emerger las clases populares como sujetos históricos luego del levantamiento de 1910, aquel concepto norteamericano de conservación como «verdadera conservación nacional» fue aún más necesario: era imperativo infundir en el pueblo el amor al árbol. La celebración del Día del Árbol, si bien ya festejada durante el porfiriato, se intensificó enormemente a partir de 1922 gracias a la intervención de la SFM.

La fama mediática de Quevedo surgió a partir de aquellos años; las mayores felicitaciones y críticas a su proyecto también. No es gratuito, pues, que el epíteto con el que se

27. El esfuerzo más sistemático se realizó sobre el pino, de enorme importancia y variedad botánica, ecológica y económica. 
le bautizó, Apóstol del árbol, reflejara ese carácter paternal, religioso y patriótico ${ }^{28}$. Carácter que inculcó en las generaciones de facultativos formados a su sombra y que, por su preparación propiamente forestal, tuvieron la oportunidad de concebir el bosque de otra manera diferente a la de su mentor, como fue el caso de Camilo del Moral.

\section{LA FORMACIÓN DE GUARDAS E INGENIEROS FORESTALES}

El cimiento absoluto de todo el proyecto de Quevedo, ya no solo como forestal, sino como servidor público, era la consideración del ingeniero como artífice de la sociedad moderna. Según él, la verdadera y única finalidad del ingeniero, su acción social, era el bienestar público, equiparable solo a la tarea realizada por el médico. Pero para eso, decía, hacía falta previsión, previsión amplia y ajustada a las necesidades del país, como la de la conservación hidrológico-forestal, que debía ser preponderante (Quevedo, 1909b: 11; 1925b: 113$117)^{29}$. Su soberbia técnica frente al ignorante de campo o de ciudad nos indica que concebía la acción social del ingeniero para la sociedad, no con ella.

La máxima de la formación forestal en México fue la de Honor, decoro y disciplina. En ella subyacía el carácter apostólico con el que Quevedo entendía la profesión, pues lo que se necesitaba era "salvar» la naturaleza de las malas costumbres ancestrales de nuestros campesinos (Quevedo, 1929: 137; 1936b: 213; Wakild, 2006: 8) ${ }^{30}$. Ahora bien, la concepción de la profesión forestal como apostolado no apareció en Quevedo sino hasta después de la Revolución. Previamente, la educación forestal operaba bajo postulados menos religiosos y más pragmáticos: vigilar, ordenar y repoblar ${ }^{31}$. Ni entonces ni después Quevedo hizo alusión a la experiencia como guía fundamental de la acción forestal.

En los inicios de este primer esfuerzo educativo e institucional destaca sobre todo la constitución del Vivero de Coyoacán (1907), como hito fundacional de los grandes objetivos de Quevedo: conservar y repoblar. En esta etapa inicial participaron un par de es-

28. El apodo le fue dado por su amigo y compañero, el ingeniero Félix Palavicini, en 1919 (SIMONIAN, 1999: 281). Por otro lado, es posible que dicho epíteto tuviera alguna relación con el Apóstol del árbol español, el famoso ingeniero de montes Ricardo Codorníu (1846-1923).

29. Por la misma razón no veía extraño que en países donde no existiera el Servicio Forestal, ni técnicos especialistas en la materia, fuera un ingeniero civil, como él, quien promoviera la implantación de aquel servicio (QUEVEDO, 1909b: 12).

30. En Alemania también se tenía la idea de que los usos tradicionales de la gente rural estaban provocando la devastación de los bosques (SANZ, 2003).

31. Junta Central de Bosques y Arbolados, El Tiempo: Diario Católico, n. ${ }^{\circ}$ 8582, 9 de junio de 1909, p. 1 . 
pañoles: un arboricultor, como jefe del vivero, y un ingeniero de montes, como inspector técnico de los bosques del valle de México. Sin embargo, como la carencia de facultativos cualificados era notoria, el propio Quevedo se pagó un viaje a Europa en abril de 1907 (con el visto bueno de la Secretaría de Fomento), para buscar personal capacitado y estudiar los métodos de plantación, la organización de viveros y las administraciones forestales de varios países de aquel continente ${ }^{32}$.

En ese viaje logró un acuerdo entre los gobiernos mexicano y francés para que este diera licencia a partir de 1908 -y por el tiempo necesario- a un grupo conformado por un botánico forestal (el ya citado G. Lapie) y siete brigadieres y guardas forestales, y para que aquel sufragara los sueldos respectivos. Ellos contribuyeron a la consolidación del proyecto forestal al participar tanto en el Vivero de Coyoacán como en la puesta en marcha de las escuelas de Coyoacán y Santa Fe (entre 1908 y 1910). La primera de ellas estaba orientada hacia la arboricultura y la segunda hacia la silvicultura. La de Coyoacán aprovechó su cercanía al vivero, mientras que la de Santa Fe la suya al bosque del Desierto de los Leones y a las zonas deforestadas del suroeste de la Ciudad de México. Un aspecto clave para escoger personal francés era porque estaban formados bajo una educación militar (pertenecían incluso al ejército), lo que los hacía, según el propio Quevedo, personas útiles y muy sumisas ${ }^{33}$.

No sin controversias, la instrucción militar fue una constante en las escuelas forestales en las que Quevedo estuvo implicado (Wakild, 2011:33-34). En su opinión ello debía ser así, porque se lograría de manera más eficaz la conservación y también porque los forestales debían ser auxiliares de la tropa, ya que los bosques constituyen un necesario elemento de defensa natural de las mismas fuerzas en campaña (Quevedo, 1943: 72).

Los forestales franceses dejaron el país en 1914, tanto por el desarrollo de la revolución en el sur de la Ciudad de México como por el estallido de la Gran Guerra (como miembros del ejército fueron llamados a filas). Quevedo lo había hecho el año anterior por las amenazas directas hacia su persona del entonces presidente Victoriano Huerta. Hasta ese momento ambas escuelas habían funcionado con normalidad. Cuando en 1914 cerró la de Santa Fe, contaba con 32 alumnos, mientras que la de Coyoacán, al momento de su clausura en diciembre de 1913, tenía solo nueve. La formación forestal se retomó

32. CEHM, archivo Limantour, fondo CDLIV, 2. ${ }^{a}$ 1907, carpeta 21, legajo 243. Correspondencia entre Miguel Ángel de Quevedo y José Y. Limantour, 30 de marzo de 1907.

33. CEHM, archivo Limantour, fondo CDLIV, 2. ${ }^{\text {a }}$ 1907, carpeta 20, legajo 191; carpeta 25, legajo 246; carpeta 24, legajo 57. Correspondencia entre Miguel Ángel de Quevedo y JoséY. Limantour: 11 de marzo de 1907, 12 de septiembre de 1907 y 10 de julio de 1910, respectivamente. BAROJAS (1937: 118); Quevedo (1943: 45-46); GonZÁlez PÉrez (2001: 348-358); VARgas (2017: 23-25). 
durante el gobierno de Venustiano Carranza por mediación e insistencia de Pastor Rouaix. En 1917 se reabrió únicamente la Escuela de Coyoacán, donde se ofertaron tres perfiles profesionales: el primero, en un año, de arboricultor y jardinero; el segundo, en dos, de perito en parques y jardines; y el tercero, en tres, de perito forestal y en parques y jardines ${ }^{34}$.

Paradójicamente, luego de la promulgación de la primera ley forestal del país en 1926, donde se exponía en el artículo 1 que el Estado se responsabilizaba de la educación y organización del personal facultativo, una disposición gubernamental del mismo año ordenó la supresión de la Escuela Forestal de Coyoacán por motivos de orden económico. Desde 1927 la preparación de forestales se trasladó a la $\mathrm{ENA}^{35}$. Para entonces, esta escuela ya estaba en sus instalaciones de Chapingo, al noreste de la Ciudad de México, donde la carrera forestal quedó reducida a su mínima expresión con tan solo un profesor para que los diez alumnos que cursaban el tercer año en Coyoacán los concluyeran ahí (Quevedo, 1927: 73).

No obstante, por iniciativa de la Universidad Nacional, la Secretaría de Educación Pública acordó instituir la carrera de ingeniero forestal a partir de 1929 en la Facultad de Ingenieros. Según Quevedo (1929: 139), porque se comprendió la necesidad absoluta de esos estudios y de la profesión, aunque pocos años después achacaría el retroceso en materia forestal para cumplir las resoluciones del Congreso de 1909 a la indiferencia del elemento universitario y educativo en general respecto de los conocimientos de la Silvicultura, ciencia fundamental de bienestar público como protectora de la Naturaleza (Quevedo, 1934: 107).

Como haya sido, la ENA empezó a ofertar la carrera de ingeniero agrónomo con especialidad en bosques a partir de 1933. Así, en la Universidad Nacional se aprovechó la estructura ya establecida para ofertar otras ingenierías, lo que reducía gastos al poder ofrecer asignaturas comunes con la forestal -que a la postre significó una tendencia hacia lo técnico y mecánico-, retribuyendo a cambio con las enseñanzas silvícolas que aquellas re-

34. GonZÁlez PÉrez (2001: 358-362); WAKILd (2006: 8); Boyer (2007: 103); Urquiza (2014: 245); VARGAS (2017: 35-36). Quienes más han estudiado el tema de las escuelas forestales entre 1908 y 1918 son GonZÁlez PÉREZ (2001) y VARGas (2017). Ambos coinciden en que la formación tenía un componente práctico muy relevante, materializado en ejercicios en el vivero, visitas a museos de historia natural, expediciones botánicas y de repoblación y ordenamiento, viajes de estudio y reconocimiento a diferentes partes del país, así como visitas a industrias forestales.

35. Algunos intentos por impartir asignaturas forestales en la ENA sucedieron en 1879, para arboricultura, en 1893, para silvicultura, dasometría y repoblación forestal, y en 1908, para dasonomía. Por falta de profesores, estas asignaturas no prosperaron (GARCía MARTínEZ, 1942: 238; EsTÉVEZ, 1956: 144; TORTOLERO, 1995: 62-63, 71, 74). 
querían también -que en la práctica se redujo a la impartición de hidrología forestal por parte de Quevedo-, mientras que en la ENA se inició la carrera sin profesores a tiempo completo, con un currículo fundamentalmente teórico y con un balance entre las asignaturas agronómicas y forestales de tres a una ${ }^{36}$.

Ambas instituciones estimaban a la forestal como una carrera menor con relación a las otras ingenierías. Así pues, en opinión de Quevedo, solo el Departamento Forestal podría impartir la formación para atender los verdaderos objetivos de la profesión, mientras que los egresados de la ENA y de la Universidad podrían ocuparse de las fincas particulares y especializarse en determinadas ramas. Sin embargo, por acuerdo de la Universidad Nacional, la carrera forestal se suspendió en 1934 (al igual que la asignatura de hidrología forestal en el resto de las ingenierías) por considerarse que la ENA cubría esa rama del conocimiento profesional (Quevedo, 1933: 197-198; 1934: 109).

La llegada de Lázaro Cárdenas a la presidencia cambió radicalmente la atención a la cuestión forestal. En enero de 1935 se creó el ya citado Departamento Autónomo Forestal y de Caza y Pesca, y en diciembre del mismo año se fundaron el Instituto de Enseñanza Superior Forestal, el Instituto de Investigaciones Forestales y la Escuela de Guardas Forestales de Tlalpan, al sur de la Ciudad de México ${ }^{37}$.

$\mathrm{Al}$ principio la nueva ingeniería impartida en el Instituto de Enseñanza comprendía tres años de estudio silvícola combinado con asignaturas cinegéticas y piscícolas. Para 1937, el plan de estudios aumentó a cuatro años al considerarse necesario fortalecer la formación científica en matemáticas, física, química, botánica, zoología y geología. Sin embargo, la carencia de personal especializado dentro de México era aún un problema; es decir, la presencia de forestales extranjeros todavía era necesaria. Igualmente hubo intentos, no sabemos si todos fructíferos, de enviar estudiantes a otros países, principalmente de Europa, pero también del continente americano (en 1926 cinco fueron a los Estados Unidos $)^{38}$.

36. RoldÁn (1929: 133); Quevedo (1929: 139-142); MARTíneZ (1967: 220-221); Borgo (1998: 253); Historia División de Ciencias Forestales (DICIFO). http://www.anech-chapingo.org.mx/histo_ bosques.html

37. La propuesta inicial era fundar tres escuelas de guardas: una en zona tropical, otra en semitropical y la última en el altiplano, que fue la que efectivamente se fundó (QUEVEDO, 1933: 197). En este sentido, cabe apuntar que no solo las carencias presupuestarias fueron motivo para crear una sola, también el hecho de que el conocimiento sobre bosques tropicales era prácticamente nulo dentro y fuera de México.

38. García Díaz (1926: 314); Quevedo (1933: 197-198; 1936a: 209); Barojas (1937: 121). 
Además de incentivar a los mejores alumnos con estancias fuera del país, también se les motivaba facilitando la continuación de sus estudios en el Instituto de Investigaciones. En este sentido, tanto Quevedo, en su calidad de jefe del Departamento Autónomo Forestal, como Ángel Roldán, en la suya de director del Instituto de Enseñanza, mostraban un futuro francamente halagüeño para quienes decidieran formar parte del gremio. De entrada, los egresados tenían asegurado un puesto en el Servicio Forestal (obligatorio por al menos tres años luego de graduarse). Pero también serían de los más queridos y respetables en la aplicación de su acción profesional por el acento práctico dado a su formación.

Sin embargo, si a principios de los años treinta había en México menos de 100 forestales, diez años después su número no superaba los 300 (cuando lo deseable era haber formado 200 por año). Se trataba de una cantidad absolutamente insuficiente para los 40 millones de hectáreas de zonas forestales estimados en el país ${ }^{39}$.

Este franco fracaso en la formación de personal contrastó con el decidido apoyo que el gobierno cardenista había otorgado a la causa forestal, cuando por fin el Estado mexicano mostraba un rostro distinto al ocuparse de movilizar los recursos naturales y humanos del país, como aseveran Boyer y Wakild (2012). A diferencia de los períodos presidenciales anteriores, en los que el propio Quevedo debía estar solicitando y negociando la atención al asunto, fue el mismo Cárdenas quien lo buscó para dirigir la gestión de los bosques, aun cuando era reconocido su pasado porfirista.Y aunque para Wakild (2006: 12) la supervivencia de Quevedo en el cardenismo se debió a la consistencia de su mensaje, a mi parecer, como la misma historiadora señalaría años después, su permanencia fue gracias al hecho de que en los años treinta seguía sin haber nadie con el reconocimiento público suficiente para hacerse cargo de la responsabilidad de dirigir un proyecto federal de política forestal (Wakild, 2011:33).

Sin embargo, los años de Quevedo al frente del proyecto forestal mexicano durante el cardenismo terminaron antes de lo esperado. En 1939 se decidió trasladar el Instituto de Enseñanza Forestal a Perote (Veracruz), y desmantelar el Instituto de Investigaciones. Aunque el golpe definitivo fue clausurar el Departamento Autónomo Forestal y de Caza y Pesca a partir del 1 de enero de 1940, y reconvertirlo -como en 1910- en una dependencia subordinada a la Secretaría de Agricultura. En sus memorias, Quevedo (1943: 84) sostiene que, aunque se dijo que era por razones presupuestarias, en realidad se trató de presiones de grupos interesados en la explotación las que condujeron a Cárdenas a suprimir el Departamento. Como haya sido, esta decisión presidencial significó el fin de la

39. Quevedo (1933: 198; 1936a: 211); RoldÁN (1936: 211-213); QUEVEDO y ROLDÁN (1937: 161); BORGO (1998: 252). 
carrera política de Quevedo y el cierre definitivo del Instituto de Enseñanza de Perote y de la Escuela Forestal de Tlalpan. La ENA quedó como la única institución universitaria donde se ofertaron conocimientos sobre bosques.

\section{CONCLUSIONES}

La irrupción de Miguel Ángel de Quevedo en la esfera pública mexicana del primer tercio del siglo Xx canalizó las aún ambiguas atenciones del Estado hacia los bosques. Al enarbolar el estandarte de la conservación forestal mediante métodos científicos, el discurso de Quevedo, novedoso y seguro de sí mismo, no solo encontró un hueco en regímenes tan dispares como el de Porfirio Díaz y el de Lázaro Cárdenas, sino que hizo de la cuestión forestal un asunto de Estado. Sin embargo, a pesar de tan enorme logro político en medio de una deforestación endémica y de un agrarismo dominante, Quevedo no supo conservar institucionalmente su proyecto en ninguno de los períodos gubernamentales en los que estuvo al frente de dependencias forestales. Lo que queda claro es que sus tácticas para introducir el método científico fueron modeladas por el clima sociopolítico de antes y después de la Revolución. Así, el impulso inicial para conformar las bases de la práctica silvícola derivó en una defensa a ultranza del árbol, cuyos guardianes, los peritos forestales, debían proteger con celo apostólico. Por tanto, las críticas de Lenz, Beltrán y Del Moral fueron al proyecto forestal posrevolucionario, cuando sin sospecharlo siquiera, Quevedo propició que los árboles escondieran al bosque. Quizá de haber prosperado la formación de ingenieros de montes en 1910 o el Instituto de Enseñanza Superior Forestal en 1940 la historia podría haber sido distinta. Aunque tampoco se debe olvidar que la marcada inclinación conservacionista de Quevedo, su indigencia conceptual, su escaso legado científico en silvicultura y su franca inclinación hacia la hidrología forestal, contribuyeron indirectamente a fundamentar los reproches a su proyecto. Como sea, no se puede dejar de reconocer la intervención del ingeniero tapatío para convertir al árbol en un elemento vital de la esfera pública, especialmente de la Ciudad de México, ni tampoco su responsabilidad en la introducción de una ciencia desconocida en el país.

\section{AGRADECIMIENTOS}

La presente investigación fue posible gracias al programa de becas posdoctorales de la Universidad Nacional Autónoma de México. El apoyo académico, moral y material que recibí en el Instituto de Geografía de dicha institución, así como de mi asesor en la investigación, Gustavo G. Garza Merodio, fue crucial para llevar a buen puerto este trabajo. Finalmente, quisiera expresar mi enorme agradecimiento a los evaluadores anónimos y 
a los revisores de Historia Agraria. Sin ninguna duda, ellos contribuyeron a mejorar sustancialmente este artículo. A todos, muchas gracias.

\section{REFERENCIAS}

ANóNimo (1897). Influencia de los bosques sobre el clima. La Naturaleza, 2. ${ }^{a}$ serie, t. II, apéndice, 35-36.

Agnoletti, M. (2006). Man, Forestry, and Forest Landscapes: Trends and Perspectives in the Evolution of Forestry and Woodland History Research. Schweizerische Zeitschrift fur Forstwesen, 157 (9), 382-392.

BÁRCENA, M. (1892). Selvicultura: Breves consideraciones sobre explotación y formación de los bosques. México: Oficina de la Secretaría de Fomento.

BAROJAS, S. (1937). Importancia de la educación forestal en el continente americano. Boletín del Departamento Forestal y de Caza y Pesca, III (9), 113-122.

BARTHOD, C. (2015). Redécouvrir Gifford Pinchot (1865-1946). Revue forestière française, 67 (1), 53-75.

BeLtRÁn, E. (1964). La batalla forestal: Lo hecho, lo no hecho, lo por hacer. México: Cultura.

Borgo, G. (Coord.) (1998). México forestal:Visto por trece profesionales del ramo. Morelia: [s. n.].

Boyer, C. (2005). Contested Terrain: Forestry Regimes and Community Responses in Northeastern Michoacán, 1940-2000. En D. B. BRAY, L. MERINO-PÉREZ \& D. BARRY (Eds.), The Community Forests of Mexico: Managing for Sustainable Landscapes (pp. 27-48). Austin: University of Texas Press.

BOYER, C. R. (2007). Revolución y paternalismo ecológico: Miguel Ángel de Quevedo y la política forestal en México, 1926-1940. Historia Mexicana, 57 (1), 91-138.

Boyer, C. R. (2015). Political Landscapes: Forests, Communities, and Conservation in Mexico. Durham: Duke University Press.

BOYER, C. R. \& WAKILD, E. (2012). Social Landscaping in the Forests of Mexico: An Environmental Interpretation of Cardenismo, 1934-1940. Hispanic American Historical Review, 92 (1), 73-106.

Caballero, M. (2000). La actividad forestal en México. Vol. II. Chapingo: Universidad Autónoma de Chapingo.

CÁrdenas, L. (1935). Mensaje del C. Presidente de la República, General Lázaro Cárdenas, radiado al pueblo mexicano el $1^{\circ}$ de enero de 1935, en lo concerniente a la creación del Departamento Autónomo Forestal y de Caza y Pesca. México Forestal, (13), $1-2$. 
CASALS, V. (1995). La formación científica, la actividad espacial y la proyección intelectual de los ingenieros de montes en la España contemporánea, 1848-1936. Tesis de grado. Barcelona: Universidad Autónoma de Barcelona.

Compañía de FÁbricas de Papel de San Rafael y ANeXas (1942). La conservación de los bosques debe tener como finalidad principal abastecer de materias primas a las industrias nacionales. Memoria de la Primera Convención Nacional Forestal (pp. 225-231). México: Secretaría Agrícola y Forestal/Dirección General Forestal y de Caza.

Delgado, J. L. (2015). Del bosque a la fábrica: Técnica y ciencia de la resina de pino en la España contemporánea. Tesis de grado. Madrid: Universidad Autónoma de Madrid.

Esteban, A \& Alfaro, A. (Dirs.) (1855). Diccionario de Agricultura Práctica y Economía Rural. Madrid: Impr. de Antonio Pérez Dubrull.

Estévez, A. (1956). Política y legislación forestal en México. Tesis de licenciatura. México: Universidad Nacional Autónoma de México.

García Díaz, E. (1926). La legislación forestal en Méjico. Revista de Montes, (I), 309314.

García Díaz, E. (1930). El esfuerzo forestal de la Compañía de las Fábricas de Papel de San Rafael y Anexas, S. A. México Forestal, vIII (11-12), 277-282.

García MartíneZ, J. (1942). La legislación forestal como base de una mejor administración de los recursos naturales de los bosques de nuestro país. En Memoria de la Primera Convención Nacional Forestal (pp. 233-241). México: Secretaría Agrícola y Forestal/Dirección General Forestal y de Caza.

GonzÁlez PÉREZ, A. (2001). La fundación de la primera escuela forestal en México. En D. PIÑERA (Coord.), La Educación superior en el proceso histórico de México (t. 2, pp. 346-362). Mexicali: Secretaría de Educación Pública/Universidad Autónoma de Baja California/Asociación Nacional de Universidades e Instituciones de Educación Superior.

GonZÁLEZ-DonCEL, I. \& GIL, L. (2013). ¿Selvicultura o silvicultura? Historia de dos palabras. En Actas $6{ }^{\circ}$ Congreso Forestal Español.Vitoria-Gasteiz, 10-14 de junio.

Guinier, PH. (1935). La enseñanza en la Escuela de Aguas y Bosques y la carrera forestal. Boletín del Departamento Autónomo Forestal y de Caza y Pesca, I (1), 195-203.

Hickel, R. (1910). L'idée forestière au Mexique. Revue des eaux et forêts, (48), 148-150. Hickel, R. (1913). Bibliographie. Revue des eaux et forêts, (52), 14-15.

KLooster, D. (2003). Campesinos and Mexican Forest Policy during the Twentieth Century. Latin American Research Review, 38 (2), 94-126.

LAPIE, G. (1912). Notes forestières d'Amérique: Mexique. Revue des eaux et forêts, (51), 619-624.

Lenz, H. (1990). Historia del papel en México y cosas relacionadas, 1525-1950. México: Miguel Ángel Porrúa. 
Martínez, F. (1967). La urgencia de mejorar la enseñanza forestal en México. En Memoria de la III Convención Nacional Forestal (pp. 225-227). Ciudad de México, 9-13 de agosto.

Mathews, A. S. (2011). Instituting Nature: Authority, Expertise, and Power in Mexican Forests. Cambridge: Massachusetts Institute of Technology Press.

Meyer, A. (1942). La silvicultura en la República Mexicana. México Forestal, xx (7-89), 53-60.

Oosthoek, J. K. \& HölzL, R. (Eds.) (2018). Managing Northern Europe's Forests: Histories from the Age of Improvement to the Age of Ecology. New York: Berghahn Books. (Environment in history, 12).

Pascual, A. (1868). Sobre el vocablo: Forestal. Revista Forestal, Económica y Agrícola, (I), 17-38, 65-80, 306-316, 538-546, 631-639, 709-716.

PACHECO, L. (2017). La participación técnica y empresarial de los ingenieros en el desarrollo hidroeléctrico de Jalisco, albores del siglo xx. En IV fornadas de Historia Económica de la Asociación Mexicana de Historia Económica. Xalapa, 21-24 de febrero. Quevedo, M. A. (1909a). La cuestión forestal en México y medidas que conviene adoptar para su resolución: Informe rendido al ciudadano Secretario de Fomento en el mes de junio de 1908 por el Presidente de la funta Central de Bosques. México: Secretaría de Fomento.

Quevedo, M. A. (1909b). La Junta Central de Bosques. Revista Forestal Mexicana, I (1), 3-13.

QueVEDO, M. A. (1911). Breve reseña de los fundamentos y métodos que han servido para la formación del Catálogo Forestal de la República. Revista Forestal Mexicana, II (6), 124-131.

QuEVEDO, M. A. (1919). La geografía y la estadística en sus relaciones con el problema forestal de México. Boletín de la Sociedad Mexicana de Geografía y Estadística, 5. ${ }^{a}$ época, IX (1), 145-152.

Quevedo, M. A. (1924). El problema de la deforestación en México: Solución práctica del mismo. En VV. AA. (2010), Miguel Ángel de Quevedo: Urbanismo y medio ambiente: Escritos de 1889 a 1941 (pp. 106-112). México: Universidad Nacional Autónoma de México/Universidad Autónoma Metropolitana-Azcapotzalco. (Raíces, 6).

Quevedo, M. A. (1925a). La riqueza forestal de México y su conservación. En VV. AA. (2010), Miguel Ángel de Quevedo: Urbanismo y medio ambiente: Escritos de 1889 a 1941 (pp. 113-116). México: Universidad Nacional Autonoma de Mexico/Universidad Autonoma Metropolitana-Azcapotzalco. (Raíces, 6).

Quevedo, M. A. (1925b). La función social del ingeniero en el aprovechamiento de los recursos naturales. México Forestal, III (8-9), 111-117.

Quevedo, M. A. (1926). La preponderante influencia de la vegetación forestal en el equilibrio de las condiciones biológicas del medio adecuado para la vida humana. En VV. 
AA. (2010), Miguel Ángel de Quevedo: Urbanismo y medio ambiente: Escritos de 1889 a 1941 (pp. 126-133). México: Universidad Nacional Autónoma de México/Universidad Autónoma Metropolitana-Azcapotzalco. (Raíces, 6).

Quevedo, M. A. (1927). Necesidad de la enseñanza profesional forestal en un instituto o escuela superior especial del ramo: Exposición de razonamientos dirigida al Ciudadano Secretario de Agricultura y Fomento. México Forestal, v (7-8), 70-74.

Quevedo, M. A. (1929). La importancia y el porvenir de la carrera de ingeniero forestal. En VV. AA. (2010), Miguel Ángel de Quevedo: Urbanismo y medio ambiente: Escritos de 1889 a 1941 (pp. 134-142). México: Universidad Nacional Autónoma de México/Universidad Autonoma Metropolitana-Azcapotzalco. (Raíces, 6).

Quevedo, M. A. (1930a). Discurso de inauguración del Primer Congreso Forestal Mexicano. México Forestal, vIII (3), 31-35.

Quevedo, M. A. (1930b). La preservación de los bosques comunales e ejidales. México Forestal, VIII (4), 73-77.

Quevedo, M. A. (1933). Proyecto de Plan Sexenal 1934-40 en lo relativo a la protección forestal del territorio. México Forestal, XI (11-12), 187-199.

Quevedo, M. A. (1934). La protección a la naturaleza en México sobre la base de protección al bosque con una amplia enseñanza de la Silvicultura y de la Hidrología Forestal. México Forestal, XII (6), 105-113.

Quevedo, M. A. (1936a). El Instituto Forestal. Boletín del Departamento Autónomo y de Caza y Pesca, I (3), 209-211.

Quevedo, M. A. (1936b). Alocución del Jefe del Departamento Forestal y de Caza y Pesca, ingeniero Miguel A. de Quevedo, al iniciarse las primeras clases en el Instituto de Enseñanza Forestal y de Caza y Pesca, el 6 de mayo de 1936. Boletín del Departamento Forestal y de Caza y Pesca, I (4), 211-214.

Quevedo, M. A. (1943). Relato de mi vida. México: [s. n.].

Quevedo, M. A. \& Roldán, A. (1937). Reglamento y plan de estudios para la carrera de Ingeniero forestal y de Caza y Pesca. Boletín del Departamento Forestal y de Caza y Pesca, II (6), 157-161.

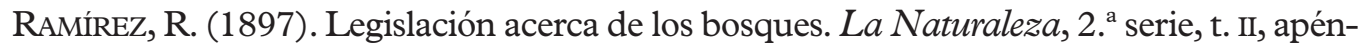
dice, 23-35.

REDACCión (1909). Por qué la Junta Central de Bosques se ha convertido en Junta Central de Bosques y Arbolados. Revista Forestal Mexicana, I (2), 29-33.

REdACCión (1939). Bibliografía forestal. Boletín del Departamento Forestal y de Caza en Pesca, IV (14), 219-231.

Reglamento para la explotación de los bosques y terrenos baldios y nacionales (1894). Ciudad de México: Secretaría de Fomento.

Roldán, A. (1929). El movimiento forestal en México. México Forestal, vII (7), 131-133. 
Roldán, A. (1936). La profesión forestal. Boletín del Departamento Autónomo y de Caza y Pesca, I (3), 211-214.

SANZ, G. (2003). Naturaleza y ciencias forestales en Alemania: Una aproximación a la historia de una tradición académica. Cuadernos de la Sociedad Española de Ciencias Forestales, (16), 161-166.

SimONIAN, L. (1999). La defensa de la tierra del jaguar: Una historia de la conservación en México. México: CONABIO/SEMARNAP/IMERNAR.

TORRES, M. (2017). Entre la búsqueda del progreso y el uso de los recursos naturales: $\mathrm{El}$ caso de la explotación de madera en México. Istor, Revista de Historia Internacional, XVIII (69), 69-83.

Tortolero, A. (1995). De la coa a la máquina de vapor: Actividad agrícola e innovación tecnológica en las haciendas de la región central de México, 1880-1914. México: Siglo XXI.

URQUIZA, J. H. (2014). Ciencia forestal, propiedad y conservación para el desarrollo nacional: Los estudios y trabajos ambientales de Miguel Ángel de Quevedo: Una historia de su influencia en las politicas de conservación de las cuencas hidrológicas (1890-1940). Tesis de grado. Ciudad de México: Universidad Nacional Autónoma de México.

VARgas, E. (2017). Los estudios científicos de Antonio H. Sosa en la conformación de los parques nacionales de México, 1935-1939. Tesis de grado. Ciudad de México: Universidad Nacional Autónoma de México.

Vera, M. R. (1903). La Dasonomía: Generalidades. México: Secretaría de Fomento.

Vergara, G. (2018). How Coal Kept My Valley Green: Forest Conservation, State Intervention, and the Transition to Fossil Fuels in Mexico. Environmental History, 23 (1), 82-105.

ViTZ, M. (2012). La ciudad y sus bosques: La conservación forestal y los campesinos en el valle de México, 1900-1950. Estudios de historia moderna y contemporánea de México, (43), 135-172.

WAKILD, E. (2006). "It is to Preserve Life, to Work for the Trees": The Steward of Mexico's Forests, Miguel Angel de Quevedo, 1862-1946. Forest History Today, 4-14.

WaKILD, E. (2011). Revolutionary Parks: Conservation, Social fustice, and Mexico's Natural Parks, 1910-1940. Tucson: University of Arizona Press. 